\title{
(2) OPEN ACCESS \\ Injury patterns and circumstances associated with electric scooter collisions: a scoping review
}

\author{
Manish Toofany, ${ }^{1}$ Sasha Mohsenian, ${ }^{2}$ Leona K Shum, ${ }^{3}$ Herbert Chan, ${ }^{3,4}$ \\ Jeffrey R Brubacher (10 3,4
}

- Additional material is published online only. To view, please visit the journal online (http://dx.doi.org/10.1136/ injuryprev-2020-044085).

${ }^{1}$ Faculty of Medicine, The University of British Columbia, Vancouver, British Columbia, Canada

${ }^{2}$ Faculty of Science, The University of British Columbia, Vancouver, British Columbia,

Canada

${ }^{3}$ Department of Emergency

Medicine, Faculty of Medicine, The University of British

Columbia, Vancouver, British

Columbia, Canada

${ }^{4}$ Centre for Clinical

Epidemiology \& Evaluation

(C2E2), Vancouver, British

Columbia, Canada

\section{Correspondence to}

Dr Jeffrey R Brubacher

Department of Emergency

Medicine, Faculty of Medicine,

The University of British

Columbia, Vancouver, BC, V6T

1Z4, Canada; jeff.brubacher@

ubc.ca

Received 15 November 2020

Revised 22 February 2021

Accepted 24 February 2021

\begin{abstract}
Background Electric scooters are personal mobility devices that have risen in popularity worldwide since 2017. Emerging reports suggest that both riders and other road users, such as pedestrians and cyclists, have been injured in electric scooter-associated incidents. We undertook a scoping review of the current literature to evaluate the injury patterns and circumstances of electric scooter-associated injuries.

Methods A scoping review of literature published from 2010 to 2020 was undertaken following accepted guidelines. Relevant articles were identified in Medline, Embase, SafetyLit and Transport Research International Documentation using terms related to electric scooters, injuries and incident circumstances. Supplemental searches were conducted to identify relevant grey literature (non-peer-reviewed reports).
\end{abstract}

Results Twenty-eight peer-reviewed studies and nine grey literature records were included in the review. The current literature surrounding electric scooterassociated injuries mainly comprises retrospective case series reporting clinical variables. Factors relating to injury circumstances are inconsistently reported. Findings suggest that the head, upper extremities and lower extremities are particularly vulnerable in electric scooter falls or collisions, while injuries to the chest and abdomen are less common. Injury severity was inconsistently reported, but most reported injuries were minor. Low rates of helmet use among electric scooter users were noted in several studies.

Conclusion Electric scooters leave riders vulnerable to traumatic injuries of varying severity. Future work should prospectively collect standardised data that include information on the context of the injury event and key clinical variables. Research on interventions to prevent electric scooter injuries is also needed to address this growing area of concern.

\section{INTRODUCTION}

Electric scooters are personal mobility devices that have been adopted as a convenient, environmentally friendly alternative to traditional modes of inner-city transportation. These scooters are typically comprised of a shaft that connects handlebars to a thin metal deck with two wheels, leaving riders only a few inches from the ground. ${ }^{1}$ Electric scooters can reach speeds up to $25 \mathrm{~km} /$ hour which allow the rider to travel on roadways or bicycle lanes. Conversely, the compact size of electric scooters also allows riders to easily manoeuvre through pedestrian traffic. ${ }^{2}$ Thus, electric scooter riders can switch between different types of road infrastructure, leaving them and other road users, including cyclists and pedestrians, vulnerable to traumatic injuries in the case of a collision.

The incidence of electric scooter-associated injuries has increased considerably since the expansion of electric scooter sharing companies in late $2017 .{ }^{3}$ So far, research on electric scooter injuries has been conducted in major urban areas across Europe, Asia and Oceania. ${ }^{4}$ In some emergency departments, the number of injuries associated with electric scooters is now similar to that of cycling injuries. ${ }^{5}$ Legislators have struggled to adapt road safety regulations to mitigate injuries due to the recent influx of electric scooters. Some jurisdictions have mandated use of protective equipment such as helmets. ${ }^{6}$ Additional evidence on injury patterns and circumstances associated with electric scooter collisions is needed to guide clinical management and inform development of policy and interventions that target modifiable risk factors for these events.

In this study, we undertook a scoping review of the current literature on electric scooter-related injuries to evaluate injury patterns, circumstances and outcomes. With evidence pertaining to trauma associated with electric scooters still emerging, this review aimed to identify gaps in the current body of literature and suggest areas for further investigation.

\section{METHODS}

This scoping review was conducted according to the Preferred Reporting Items for Systematic Reviews and Meta-Analyses extension for Scoping Reviews methodology. ${ }^{7}$ Our search protocol was published in the Open Science Framework (https:/osf.io/ fp5uv) prior to study commencement.

\section{Search methodology}

A systematic search of English-language peerreviewed studies was conducted using four databases: Medline, Embase, SafetyLit, and the Transport Research International Documentation (TRID). The TRID database also includes nonpeer reviewed 'grey literature' such as reports (see below). As the popularity of electric scooters is fairly recent, the search was limited to articles published between January 2010 and December 2020. Keywords and/or subject headings were used to define three concepts (electric scooter, injury and circumstances) as per online supplemental table 6 .

The first two concepts (electric scooter and injury) were combined to examine injury patterns specifically regarding severity, type and location of injury. The first and third concepts (electric 
scooter and circumstances) were combined to examine the injury circumstances specifically regarding the street/road environment, motor vehicle traffic and causes of the events. A sample search is included in online supplemental table 7. References from selected articles were examined to look for relevant articles that were not included in the database search.

\section{Inclusion criteria}

To be included, studies and documents needed to: (1) examine the injuries of patients following electric scooter-associated injury events; (2) have electric scooter-specific data; (3) have electric scooter-associated injury events comprise at least 25\% of the study sample; and (4) be published between January 2010 and December 2020.

\section{Exclusion criteria}

Studies and documents were excluded if: (1) only injuries requiring a specific medical treatment were included; (2) only the treatment or management of injuries was reported; (3) case series included fewer than 20 cases; or (4) studies were reported as an abstract only.

\section{Risk of bias (quality) assessment}

The quality of studies was independently assessed by two reviewers using the National Heart Lung, and Blood Institute (NHLBI) assessment tools for evaluating observational studies. The specific tool applied was chosen based on study design. ${ }^{8}$ This ensured that the internal validity and risk of bias of each study were assessed in a similar manner irrespective of study design. Disagreements were resolved through discussions between two reviewers (MT, SM) and by consulting a third reviewer when needed.

\section{Grey literature search}

Grey literature was identified through a TRID search, a Google Scholar search, a general Google search and a Google custom search. The Google custom search focused on government documents. In all cases, the following terms: ("Scooter" or "Micromobility", or "Personal Mobility") and ("Injury" or "Trauma” or "Accident") were used to search for exact terms. Searches were conducted up to 31 December 2020.

\section{Data extraction}

Two reviewers (MT, SM) independently screened titles and abstracts for eligible articles from the initial search. For articles that were unclear for eligibility, consensus was made through discussions among all team members. The selected articles were then reviewed in detail using standardised inclusion and exclusion criteria by MT and SM. Disagreements were resolved through discussions among all team members. Inter-rater reliability was measured with Cohen's kappa coefficient. For each included study, the following data were independently extracted by the two reviewers: study title, first author, year of publication, data collection period, study aims, study methodology, country, participant demographics, sample road user type (ie, electric scooter driver, non-driver), injury distribution, injury type, injury rate, injury severity, clinical care in emergency department, emergency department disposition, hospital admission length, mechanism of injury, collision road type, helmet use and substance use.

\section{Analysis}

Due to heterogeneity in the data reported, narrative syntheses were used to summarise principal findings. Descriptive statistics were performed when possible for key data categories that were reported using similar metrics across numerous studies.

\section{RESULTS}

\section{Search results}

The search strategy yielded 614 unique records. Twenty-eight peer-reviewed studies and nine grey literature records were included in the final review following the title, abstract and full-text eligibility review (figure 1). Cohen's Kappa coefficient between the two reviewers (MT and SM) was 0.83. There were no disagreements between the two reviewers in the quality assessment of selected studies.

\section{Peer-reviewed studies}

Twenty-eight peer-reviewed studies were included, of which 25 were retrospective case series and three were prospective observational studies. No scoping or systematic reviews related to electric scooter trauma were identified. Twenty-three studies were conducted in urban regional trauma centres: 11 single-site studies and 12 multisite. Of these studies, 13 were conducted in the USA, 3 in New Zealand and 1 each in Singapore, Australia, Denmark, France, Finland, South Korea and Germany. Four nationwide studies were conducted in the USA through analyses of the United States Consumer Product Safety Commission National Electronic Injury Surveillance System (NEISS). These four studies were counted as a single study when reporting injury patterns and circumstances in the following sections as they used the same database. Additionally, one study employed information from a city-wide database in the USA. Publication dates ranged from 2019 to 2020 . No included studies were published between 2010 and 2018. The NHLBI assessment of the included studies ranged from 'Fair' to 'Good'. A summary of the study designs can be found in table 1 .

The majority of studies sampled patients retrospectively through emergency department or trauma services databases (table 1). All studies reported electric scooter riders as part of their sample, eight studies also reported non-riders injured in an electric scooter collision. Data collection (table 1) was performed through retrospective medical chart reviews for 20 studies. Four studies used NEISS data. Four studies were based on interviews with injured electric scooter riders, including one study that conducted interviews during participants' emergency department visits.

The most frequently reported aspects relating to injury patterns were injury distribution $(n=28)$, injury type $(n=28)$ and emergency department disposition $(n=19)$. Emergency department procedures $(n=10)$, injury rate $(n=9)$ and surgical procedures $(n=7)$ were also commonly reported.

The most frequently reported factors relating to injury circumstances were helmet use $(n=21)$, alcohol use $(n=18)$ and mechanism of injury $(n=13)$. The location of the event (eg, road, sidewalk, bike path) was only reported in four studies.

\section{Injury patterns}

The distribution (table 2) of electric scooter-associated injuries was reported in 25 separate studies (counting the NEISS studies as one study), but the categorisation of injuries varied between studies. Thirteen studies categorised all injuries into the same six body regions (ie, head, face, chest, upper extremity, abdomen and lower extremity). ${ }^{349-19}$ In these studies, the most commonly injured regions were the upper extremity (one of three most frequently injured regions in 12 studies), head (11 studies) and lower extremity (10 studies) whereas the chest and abdomen 


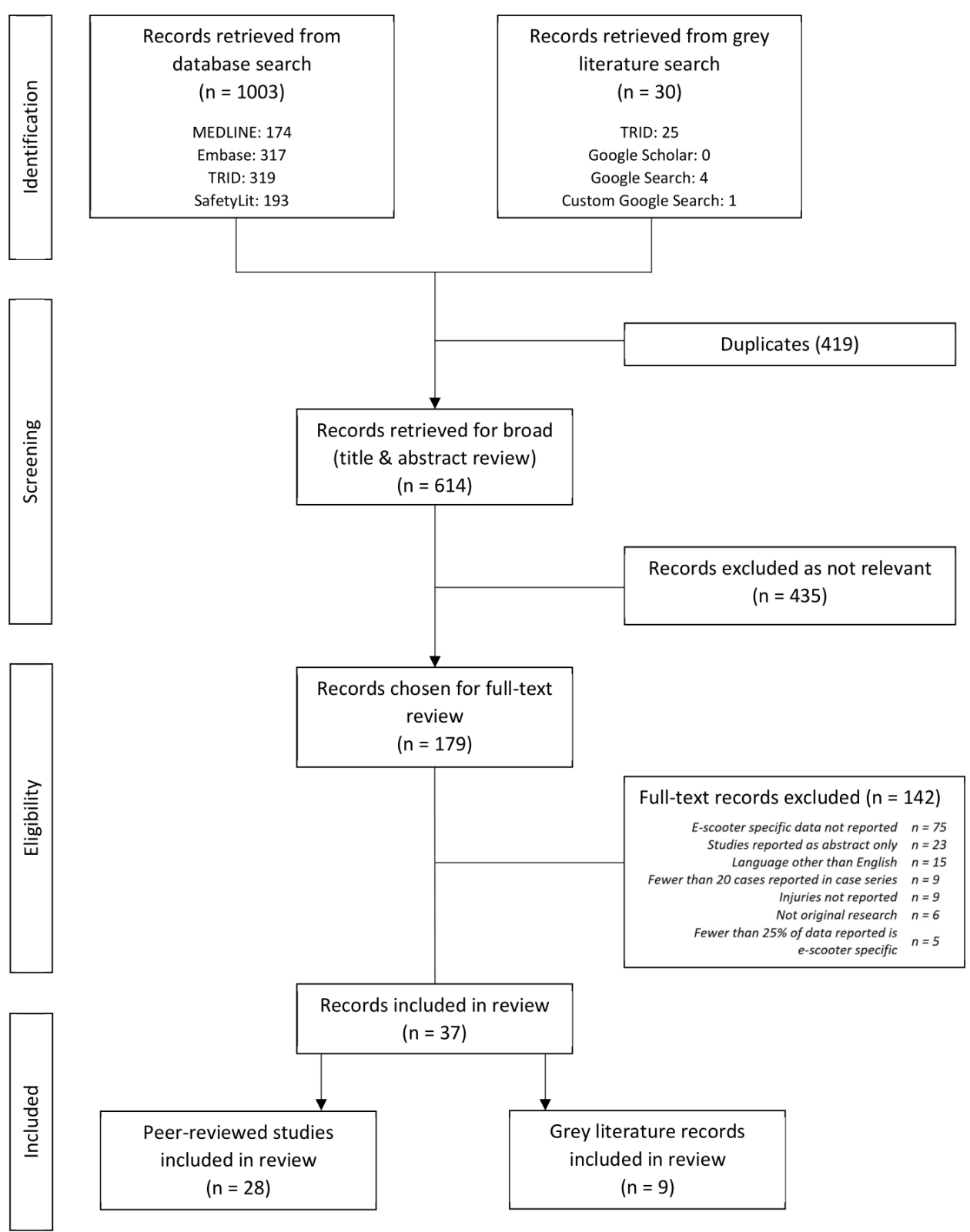

Figure 1 Preferred Reporting Items for Systematic Reviews and Meta-Analyses search decision flow chart. TRID, Transport Research International Documentation.

were the least frequently injured regions (table 2). Eight studies looked specifically at craniofacial injuries and all reported that the upper and mid regions of the face as well as the orbit were especially vulnerable to injuries (table 2). Dental injuries were also noted as being common in patients with craniofacial injuries. $^{19-26}$

Ten studies included all patients with electric scooter-associated trauma and reported the number of patients who sustained fractures (table 3). ${ }^{3} 4101113-18$ Overall almost a third of all patients in these studies sustained a fracture $(341 / 1112=30.7 \%$; range: $11.5 \%-70.5 \%)$.

The median Injury Severity Score (ISS) was reported in seven studies (table 3) that assessed all electric scooter-associated trauma emergency department patients and ranged from 1.0 to 5.5.11 $12141827-29$

The number of patients who underwent imaging procedures (table 4) in the emergency department was reported in six studies. $^{51113161828}$ Two-thirds of all patients in these six studies required a procedure $(480 / 696=68.9 \%$; range: $32.6 \%-90.3 \%)$. Consistent findings between these six studies, and in two additional studies that focused on imaging, suggest that radiographs account for the majority of these procedures. ${ }^{30} 31$

As for emergency department disposition (table 4), data from 13 studies ( $n=2022$ participants) showed that most patients injured as a result of an electric scooter collision $(1690 / 2022=86 \%$; range 66.7\%-94.0\%) did not require hospital admission and could be treated as outpatients. ${ }^{3} 5$ 9-11 13-17 1926283032

\section{Injury circumstances}

Ten studies ( $\mathrm{n}=1214$ participants) reported mechanism of injury (table 5) in all patients with electric scooter-related trauma. 41011 15-19 2833 These studies report that 92.8\% of riders were injured in single road user events, while $7.1 \%$ were injured in multiple road user events. Single user events included falls, collisions with objects, excessive speed and unfavourable road conditions, 
Table 1 Characteristics of selected studies

\begin{tabular}{|c|c|c|c|c|c|c|c|}
\hline Study & Country & Study design & $\begin{array}{l}\text { Number of } \\
\text { sites }\end{array}$ & $\begin{array}{l}\text { Primary data } \\
\text { source }\end{array}$ & Time period & Sample size & Critical appraisal \\
\hline \multicolumn{8}{|c|}{ Peer-reviewed publications } \\
\hline Trivedi et al $2019^{16}$ & USA & Retrospective & 2 & Medical charts & September 2017-August 2018 & 249 & Good \\
\hline Störmann et al $2020^{15}$ & Germany & Prospective & 2 & $\begin{array}{l}\text { Prospective data } \\
\text { collection }\end{array}$ & July 2019-March 2020 & 76 & Good \\
\hline Mitchell et al $2019^{13}$ & Australia & Retrospective & 1 & Medical charts & November 2018-January 2019 & 54 & Good \\
\hline Liew et al $2020^{28}$ & Singapore & Retrospective & 1 & Medical charts & 2015-2016 & 36 & Good \\
\hline Kobayashi et al $2019^{12}$ & USA & Retrospective & Multiple & Medical charts & September 2017-October 2018 & 103 & Good \\
\hline Brownson et al $2019^{11}$ & New Zealand & Retrospective & 1 & Medical charts & October 2018-February 2019 & 180 & Good \\
\hline Blomberg et al $2019^{4}$ & Denmark & Retrospective & Multiple & Medical charts & January 2016-July 2019 & 130 & Good \\
\hline Beck et al 2019 & New Zealand & Retrospective & 1 & Medical charts & $\begin{array}{l}\text { January 2018-February } 2018 \text { and } \\
\text { January 2019-February } 2019\end{array}$ & 54 & Good \\
\hline Bauer et al $2020^{10}$ & USA & Retrospective & 1 & Medical charts & October 2018-October 2019 & 61 & Good \\
\hline Puzio et al $2020^{14}$ & USA & Retrospective & 2 & Medical charts & September 2018-November 2018 & 92 & Fair \\
\hline Badeau et al $2019^{32}$ & USA & Retrospective & 2 & Medical charts & $\begin{array}{l}\text { June 2017-November } 2017 \text { and } \\
\text { June 2018-November } 2018\end{array}$ & 50 & Good \\
\hline Namiri et al $2020^{3}$ & USA & Retrospective & Multiple & NEISS & 2014-2018 & 988 & Fair \\
\hline Aizpuru et al $2019^{9}$ & USA & Retrospective & Multiple & NEISS & 2013-2017 & 820 & Fair \\
\hline Bresler et al $2019^{23}$ & USA & Retrospective & Multiple & NEISS & 2008-2017 & 990 & Fair \\
\hline Farley et al $2020^{47}$ & USA & Retrospective & Multiple & NEISS & 2014-2019 & 1823 & Fair \\
\hline $\begin{array}{l}\text { Yarmohammadi et al } \\
2020^{20}\end{array}$ & USA & Retrospective & 2 & Medical charts & June 2018-May 2019 & 34 & Fair \\
\hline Faraji et al $2020^{21}$ & USA & Retrospective & 1 & Medical charts & April 2018-September 2019 & 203 & Good \\
\hline Trivedi et a/ $2019^{22}$ & USA & Retrospective & 1 & Medical charts & July 2018-January 2019 & 90 & Good \\
\hline Siow et al $2020^{29}$ & USA & Retrospective & 1 & Medical charts & November 2017-January 2020 & 486 & Good \\
\hline Ishmael et al $2020^{33}$ & USA & Retrospective & 2 & Medical charts & September 2017-August 2019 & 73 & Fair \\
\hline Dhillon et al $2020^{27}$ & USA & Retrospective & Multiple & Medical charts & January 2018-December 2018 & 87 & Good \\
\hline $\begin{array}{l}\text { Nellamattathil et al } \\
2020^{31}\end{array}$ & USA & Retrospective & Multiple & Medical charts & September 2017-December 2018 & 54 & Good \\
\hline $\begin{array}{l}\text { Mayhew and Bergin } \\
2019^{30}\end{array}$ & New Zealand & Retrospective & 1 & Medical charts & August 2018-December 2018 & 64 & Fair \\
\hline English et al $2020^{18}$ & USA & Prospective & 2 & $\begin{array}{l}\text { Interviews and } \\
\text { medical charts }\end{array}$ & September 2018-November 2018 & 124 & Good \\
\hline Hennocq et al $2020^{24}$ & France & $\begin{array}{l}\text { Prospective and } \\
\text { retrospective }\end{array}$ & 2 & $\begin{array}{l}\text { Interviews and } \\
\text { medical charts }\end{array}$ & January 2017-October 2019 & 125 & Good \\
\hline Kim et al $2020^{25}$ & South Korea & Retrospective & 1 & Medical charts & January 2017-March 2020 & 256 & Good \\
\hline Oksanen et al $2020^{26}$ & Finland & Retrospective & 1 & Medical charts & 2019 & 23 & Good \\
\hline Shiffler et al $2020^{19}$ & USA & Retrospective & Multiple & Database & 2017-2019 & 165 & Good \\
\hline \multicolumn{8}{|c|}{ Grey literature (non-journal reports) } \\
\hline Pearson et al $2019^{35}$ & Australia & Retrospective & NR & NR & November 2018-February 2019 & 82 & \\
\hline Hojjat et al $2019^{36}$ & USA & Retrospective & Multiple & NEISS & 2013-2017 & $3458^{a}$ & \\
\hline Beck et al $2020^{37}$ & New Zealand & Retrospective & 1 & Medical charts & 2018-2019 & 56 & \\
\hline $\begin{array}{l}\text { Changand Diamond } \\
2019^{38}\end{array}$ & USA & Retrospective & Multiple & NEISS & 2013-2017 & 444 & \\
\hline Allen et al $2019^{39}$ & USA & Retrospective & NR & Medical charts & September 2018-November 2018 & 200 & \\
\hline Sedor and Caswell $2019^{40}$ & Canada & NR & NR & NR & July 2019-August 2019 & 33 & \\
\hline $\begin{array}{l}\text { Austin Public Health Unit } \\
2019^{17}\end{array}$ & USA & Retrospective & NR & Interviews & September 2018-November 2019 & 192 & \\
\hline $\begin{array}{l}\text { City of Santa Monica } \\
2019^{41}\end{array}$ & USA & NR & NR & NR & January 2017-September 2019 & NR & \\
\hline Cicchino et al $2020^{34}$ & USA & Prospective & 1 & Interviews & March 2019-September 2019 & 103 & \\
\hline
\end{tabular}

NEISS, National Electronic Injury Surveillance System; NR, not reported.

with falls $(94.6 \%)$ being the most common (table 5). Five of these 10 studies included injury circumstances for non-electric scooter riders (table 5), namely pedestrians and cyclists $(n=44)$. In these five studies, the most common mechanisms of injury to non-riders included being struck by an electric scooter $(26 / 44=59.1 \%)$ and tripping over a stationary electric scooter $(13 / 44=29.5 \%) .{ }^{4514} 1828$

Data from 16 studies $(n=1656)$ showed that only $4.5 \%$ of electric scooter riders were helmeted (table 5) at the time of their injury, while $67.5 \%$ were unhelmeted, and the remaining $28.0 \%$ had unknown helmet status. ${ }^{45101113-1719222326282932}$ A protective effect of helmets on the incidence of head injuries was noted in the sole study where this association was examined. ${ }^{13}$

The median per cent of patients whose injuries were associated with alcohol use (table 5) was $26.5 \%$ (IQR 13-48) as reported across 10 studies that reported alcohol use in all electric scooter-associated trauma patients. 45 10-14 161719

The five included studies that reported the location (table 5) of the event (eg, road, sidewalk, bike path) found that streets 
Table 2 Key findings of selected literature: injury distribution

\begin{tabular}{|c|c|c|c|c|c|c|c|c|}
\hline Study & $\begin{array}{l}\text { Upper } \\
\operatorname{limb}(\%)\end{array}$ & $\begin{array}{l}\text { Lower } \\
\operatorname{limb}(\%)\end{array}$ & Head $(\%)$ & Face $(\%)$ & Chest (\%) & Abdomen (\%) & Spine (\%) & Other \\
\hline \multicolumn{9}{|c|}{ Peer-reviewed publications } \\
\hline Trivedi et al $2019^{16}$ & 18 & 6 & 40 & 6 & 2 & & 1 & \\
\hline Störmann et al $2020^{15}$ & 47 & 36 & 17 & 21 & 9 & 0 & & \\
\hline Mitchell et al $2019^{13}$ & 54 & 25 & 15 & & 1 & 6 & & \\
\hline Liew et al $2020^{28}$ & & & & 11 & 6 & & & $\begin{array}{l}\text { Extremities: } 33 \% \\
\text { External, unspecified: } 72 \%\end{array}$ \\
\hline Kobayashi et al $2019 * 12$ & 13 & 22 & & 26 & & & & \\
\hline Brownson et al $2019^{11}$ & & & 17 & 11 & 3 & 0 & & Extremities: $55 \%$ \\
\hline Blomberg et al $2019^{4}$ & 26 & 28 & 22 & 28 & 3 & & 3 & \\
\hline Beck et al 2019 * & 18 & 9 & 26 & 6 & & & & \\
\hline Bauer et al $2020^{10}$ & 16 & 18 & & & & & & $\begin{array}{l}\text { Head, face, and/or neck: } 44 \% \\
\text { Torso: } 12 \%\end{array}$ \\
\hline Puzio et al $2020^{14}$ & 36 & 17 & 18 & 12 & 1 & 3 & & Multisystem: $12 \%$ \\
\hline Badeau et al $2019^{32}$ & & & 20 & & & & & $\begin{array}{l}\text { MSK: } 70 \% \\
\text { Superficial, unspecified: } 40 \%\end{array}$ \\
\hline Namiri et al $2020^{3}$ & 26 & 32 & 32 & & & & & Torso: $10 \%$ \\
\hline Aizpuru et al $2019^{9}$ & 26 & 35 & 28 & & & & & Torso: $11 \%$ \\
\hline Dhillon et al $2020^{27}$ & & & & & 9 & & 9 & $\begin{array}{l}\text { Extremities: } 22 \% \\
\text { Craniofacial: } 23 \%\end{array}$ \\
\hline English et al $2020^{18}$ & 56 & 34 & 18 & 33 & 8 & 2 & & \\
\hline Farley et al $2020^{47}$ & & & 27 & & & & & \\
\hline Bresler et al $2019^{23}$ & & & 65 & 24 & & & 4 & $\begin{array}{l}\text { Mouth: } 7 \% \\
\text { Neck: } 6 \% \\
\text { Ear: } 1 \% \\
\text { Eye: } 1 \%\end{array}$ \\
\hline Yarmohammadi et al $2020 t^{20}$ & & & & 100 & & & & $\begin{array}{l}\text { Lateral orbital wall: } 56 \% \\
\text { Orbital floor: } 53 \% \\
\text { Orbital roof: } 28 \% \\
\text { Medial orbital wall: } 25 \% \\
\text { Jaw: } 15 \% \\
\text { Nose: } 9 \%\end{array}$ \\
\hline Faraji et al $2020 t^{21}$ & & & 11 & 50 & & & & $\begin{array}{l}\text { Mouth: } 15 \% \\
\text { Ear: } 1 \% \\
\text { Nose: } 8 \%\end{array}$ \\
\hline Trivedi et al $2019^{22}$ & & & 37 & 44 & 2 & 3 & 2 & Extremities: $64 \%$ \\
\hline Hennocq et al $2020 t^{24}$ & & & & 100 & & & & $\begin{array}{l}\text { Forehead: } 18 \% \\
\text { Nose: } 11 \% \\
\text { Cheek: } 3 \% \\
\text { Lips: } 28 \% \\
\text { Chin: } 40 \%\end{array}$ \\
\hline Kim et al $2020^{25}$ & & & & & & & & Craniofacial: $49 \%$ \\
\hline Oksanen et al $2020 t^{26}$ & & & & & 4 & & & $\begin{array}{l}\text { Midface: } 43 \% \\
\text { Mandible: } 26 \% \\
\text { Skull base: } 9 \%\end{array}$ \\
\hline Shiffler et al $2020^{19}$ & 47 & 18 & & & 1 & 0 & 0 & Craniofacial: $23 \%$ \\
\hline Siow et al $2020 \ddagger^{29}$ & 49 & 25 & & & & & 4 & Polytrauma: $10 \%$ \\
\hline Ishmael et al $2020 \ddagger^{33}$ & 44 & 58 & & & & & & \\
\hline Nellamattathil and Amber $2020^{* 31}$ & 15 & 6 & & 4 & 4 & & & \\
\hline Mayhew and Bergin $2019^{30}$ & & & & & 10 & & 3 & $\begin{array}{l}\text { Extremities: } 84 \% \\
\text { Head/face: } 44 \%\end{array}$ \\
\hline \multicolumn{9}{|c|}{ Grey literature (non-journal reports) } \\
\hline Pearson et al $2019^{35}$ & & & & & & & & $\begin{array}{l}\text { Most common: upper limb, lower limb, } \\
\text { head/neck }\end{array}$ \\
\hline \multicolumn{9}{|l|}{ Hojjat et al $2019^{36}$} \\
\hline Beck et al $2020^{37}$ & & & & & & & & $\begin{array}{l}\text { Most common: head, upper limb, } \\
\text { lower limb. }\end{array}$ \\
\hline Chang and Diamond $2019^{38}$ & & & & & & & & Most common: head/face \\
\hline Allen et al $2019^{39}$ & 56 & 36 & & & & & & Head/face: $46 \%$ \\
\hline \multicolumn{9}{|l|}{ Sedor and Caswell $2019^{40}$} \\
\hline Austin Public Health Unit $2019^{17}$ & 70 & 55 & 48 & & & & & Chest/abdomen: $18 \%$ \\
\hline \multicolumn{9}{|l|}{ City of Santa Monica $2019^{41}$} \\
\hline Cicchino et al $2020^{34}$ & 34 & 18 & 7 & 9 & & & & Chest, abdomen, or spine: $3 \%$ \\
\hline
\end{tabular}

*Reported distribution of fractures.

tReported distribution of craniofacial injuries.

¥Reported distribution of orthopaedic injuries. 


\section{Systematic review}

Table 3 Key findings of selected literature: injury type and severity

\begin{tabular}{|c|c|c|c|c|c|c|}
\hline \multirow[b]{2}{*}{ Study } & \multicolumn{4}{|c|}{ Type of injury } & \multicolumn{2}{|c|}{ Injury severity } \\
\hline & $\begin{array}{l}\text { Superficial soft } \\
\text { tissue injury (\%) }\end{array}$ & $\begin{array}{l}\text { Fracture or } \\
\text { dislocation (\%) }\end{array}$ & Head injury (\%) & Internal injury (\%) & ISS & Other \\
\hline \multicolumn{7}{|c|}{ Peer-reviewed publications } \\
\hline Trivedi et al $2019^{16}$ & 56 & 36 & 40 & 1 & & Urgent * $33 \%$ \\
\hline Störmann et al $2020^{15}$ & 58 & 42 & 17 & & & \\
\hline Mitchell et al $2019^{13}$ & 60 & 24 & 15 & & & \\
\hline Liew et al $2020^{28}$ & 72 & 33 & & & $\begin{array}{l}\text { Median } 1 \\
\text { Range 1-5 }\end{array}$ & \\
\hline Kobayashi et al $2019^{12}$ & NR & 42 & 19 & & $\begin{array}{l}\text { Median } 5.5 \\
\text { IQR 5-9 }\end{array}$ & \\
\hline Brownson et al $2019^{11}$ & 66 & 42 & 17 & & $\begin{array}{l}\text { Median } 4 \\
\text { Range 1-29 }\end{array}$ & \\
\hline Blomberg et al $2019^{4}$ & 31 & 12 & 22 & & & Urgent $^{*} 7 \%$ \\
\hline Beck et al 2019 & 46 & 32 & 26 & & & Urgent* $17 \%$ \\
\hline Bauer et al $2020^{10}$ & 54 & 33 & 17 & & $\begin{array}{l}\text { Mean } 6.3 \\
\text { SD } 6.0\end{array}$ & \\
\hline Puzio et al $2020^{14}$ & 34 & 24 & 17 & & Median 1 & \\
\hline Badeau et al $2019^{32}$ & 74 & 36 & 20 & & & \\
\hline Namiri et al $2020^{3}$ & 37 & 27 & 32 & & & \\
\hline Aizpuru et al $2019^{9}$ & 31 & 26 & 28 & & & \\
\hline Dhillon et al $2020^{27}$ & & 55 & 23 & & $\begin{array}{l}\text { Mean } 7.2 \\
\text { Median } 5 \\
\text { IQR 2-10 }\end{array}$ & \\
\hline English et al $2020^{18}$ & 65 & 33 & 18 & 1 & $\begin{array}{l}\text { Median } 5 \\
\text { IQR 4-9 }\end{array}$ & \\
\hline Farley et al $2020^{47}$ & & & 15 & 27 & & \\
\hline Bresler et al $2019^{23}$ & 32 & 5 & 36 & & & \\
\hline Yarmohammadi et al $2020 t^{20}$ & & 94 & 21 & & & \\
\hline Faraji et al $2020^{21}$ & 65 & 27 & & & & \\
\hline Trivedi et al $2019^{22}$ & 42 & & & & & Severe $\neq 58 \%$ \\
\hline Hennocq et al $2020^{24}$ & 62 & 47 & & & & \\
\hline Kim et al $2020^{25}$ & 44 & 9 & 30 & & & \\
\hline Oksanen et al $2020^{26}$ & 91 & 65 & 22 & & & \\
\hline Shiffler et al $2020^{19}$ & 20 & 23 & & & & \\
\hline Siow et al $2020 \S^{29}$ & NR & 49 & & & $\begin{array}{l}\text { Mean } 8.4 \\
\text { Median } 5.0\end{array}$ & \\
\hline Ishmael et al $2020 \S^{33}$ & NR & 93 & & & & \\
\hline Nellamattathil and Amber $2020^{31}$ & NR & 26 & & & & \\
\hline Mayhew and Bergin $2019^{30}$ & NR & 57 & 24 & & & \\
\hline \multicolumn{7}{|c|}{ Grey literature (non-journal reports) } \\
\hline \multicolumn{7}{|l|}{ Pearson et al $2019^{35}$} \\
\hline \multicolumn{7}{|l|}{ Hojjat et al $2019^{36}$} \\
\hline Beck et al $2020^{37}$ & & 32 & 26 & & & \\
\hline \multicolumn{7}{|l|}{ Chang and Diamond $2019^{38}$} \\
\hline \multicolumn{7}{|l|}{ Allen et al $2019^{39}$} \\
\hline \multicolumn{7}{|l|}{ Sedor and Caswell $2019^{40}$} \\
\hline Austin Public Health Unit $2019^{17}$ & & 35 & 15 & & & \\
\hline City of Santa Monica 2019 & & & & & & Minor injuries in $80 \%$ \\
\hline Cicchino et al $2020^{34}$ & 53 & & 7 & & & Max. AIS $\leq 2$ in $98 \%$ \\
\hline
\end{tabular}

*Urgent defined as Canadian Triage Acuity Score of 1 (resuscitation), 2 (emergent) or 3 (urgent).

tReported for patients with facial injuries.

$¥$ Severe defined as fracture, concussion or intracranial haematoma.

$\S$ Reported for patients with orthopaedic injuries.

AIS, Abbreviated Injury Scale; ISS, Injury Severity Score.

and sidewalks were most common. ${ }^{1718243134}$ Additional details of injury circumstances are summarised in online supplemental appendix table 6.

\section{Grey literature}

The grey literature search yielded five conference proceedings, three government reports, and one report from a scientific organisation. ${ }^{17}{ }^{34-41}$ The included grey literature supported the injury distribution, injury severity, mechanism of injury and low rates of helmet use reported by the peer-reviewed studies.

\section{DISCUSSION}

To the best of our knowledge, this is the first scoping review of electric scooter trauma. All 28 included peer-reviewed 
Table 4 Key findings of selected literature: resource utilisation

\begin{tabular}{|c|c|c|c|c|c|c|c|}
\hline \multirow[b]{2}{*}{ Study } & \multicolumn{4}{|c|}{ Diagnostic studies } & \multicolumn{3}{|c|}{ Hospital resources } \\
\hline & Any imaging (\%) & Radiograph (\%) & CT scan (\%) & Ultrasound (\%) & $\begin{array}{l}\text { Emergency } \\
\text { department } \\
\text { procedure (\%) }\end{array}$ & $\begin{array}{l}\text { Surgical intervention } \\
(\%)\end{array}$ & $\begin{array}{l}\text { Admission to } \\
\text { hospital (\%) }\end{array}$ \\
\hline \multicolumn{8}{|c|}{ Peer-reviewed publications } \\
\hline Trivedi et al $2019^{16}$ & 80 & & & & & & 6 \\
\hline Störmann et al $2020^{15}$ & & & & & & 28 & \\
\hline Mitchell et al $2019^{13}$ & 92 & 78 & 24 & 0 & & & 13 \\
\hline Liew et al $2020^{28}$ & 72 & 72 & 15 & & & 9 & 26 \\
\hline Kobayashi et al $2019^{12}$ & & & & & & 33 & \\
\hline Brownson et al $2019^{11}$ & 45 & & 33 & & & 22 & 20 \\
\hline \multicolumn{8}{|l|}{ Blomberg et al $2019^{4}$} \\
\hline Beck et al 2019 & 72 & 69 & 17 & & & 7 & 22 \\
\hline Bauer et al $2020^{10}$ & & & & & & & 25 \\
\hline Puzio et al $2020^{14}$ & & & & & & 24 & 9 \\
\hline Badeau et al $2019^{32}$ & & & & & & 14 & 16 \\
\hline \multicolumn{8}{|l|}{ Namiri et al $2020^{3}$} \\
\hline Aizpuru et al $2019^{9}$ & & & & & & & 9 \\
\hline Dhillon et al $2020^{27}$ & & & & & & 17 & \\
\hline English et a/ $2020^{18}$ & 90 & 71 & 40 & & 63 & 21 & 28 \\
\hline Farley et al $2020^{47}$ & & & & & & & 8 \\
\hline \multicolumn{8}{|l|}{ Bresler et al $2019^{23}$} \\
\hline Yarmohammadi et al $2020^{20}$ & & & & & & 24 & 76 \\
\hline \multicolumn{8}{|l|}{ Faraji et al $2020^{21}$} \\
\hline Trivedi et al $2019^{22}$ & & & & & & & 23 \\
\hline \multicolumn{8}{|l|}{ Hennocq et al $2020^{24}$} \\
\hline \multicolumn{8}{|l|}{ Kim et al $2020^{25}$} \\
\hline Oksanen et al $2020^{26}$ & & & & & & & 61 \\
\hline Shiffler et a/ $2020^{19}$ & & & & & & & 21 \\
\hline Siow et al $2020^{29}$ & & & & & & 26 & 37 \\
\hline \multicolumn{8}{|l|}{ Ishmael et al $2020^{33}$} \\
\hline Nellamattathil and Amber $2020^{\star 31}$ & 100 & 83 & 15 & 0 & & & \\
\hline Mayhew and Bergin 2019*30 & 100 & 82 & 18 & 0 & & 25 & 40 \\
\hline \multicolumn{8}{|c|}{ Grey literature (non-journal reports) } \\
\hline Pearson et al $2019^{35}$ & 87 & & & & & & \\
\hline \multicolumn{8}{|l|}{ Hojjat et al $2019^{36}$} \\
\hline Beck et al $2020^{37}$ & 65 & & & & & 9 & 20 \\
\hline \multicolumn{8}{|l|}{ Chang and Diamond $2019^{38}$} \\
\hline Allen et al $2019^{39}$ & 91 & & & & 61 & 21 & 29 \\
\hline \multicolumn{8}{|l|}{ Sedor and Caswell $2019^{40}$} \\
\hline Austin Public Health Unit $2019^{17}$ & & & & & & & 15 \\
\hline \multicolumn{8}{|l|}{ City of Santa Monica 2019} \\
\hline Cicchino et al $2020^{34}$ & & & & & $\begin{array}{l}\text { Splinting: } 48 \\
\text { Wound care: } 35 \\
\text { Laceration } \\
\text { repair: } 24\end{array}$ & 12 & 9 \\
\hline
\end{tabular}

* Reported only for patients who required imaging during their visit.

studies were published between 2019 and 2020, which suggests research in this area is recent and may increase rapidly in the coming years. The recent literature may be in response to the recent proliferation of shared electric scooter schemes leading to increases in related emergency department visits. ${ }^{3}$

The most commonly injured body parts following electric scooter-associated trauma were the head, upper extremities and lower extremities as expected. The chest and abdomen were consistently the two least common injured regions. This injury pattern is similar to that observed in certain non-motorised mobility devices such as skateboards and non-motorised scooters. ${ }^{42}$ However, electric scooter injuries are likely more severe due to their increased speeds, as noted in two included studies. ${ }^{44}$ Falls were the leading cause of injuries in electric scooter riders, and many upper extremity injuries result from falls on an outstretched hand, a common reaction used to break the impact of a fall. ${ }^{43}$ Electric scooters' low height off the ground along with riders' reflex to step off the scooter in risky situations may explain the high frequency of lower extremity injuries. ${ }^{1}$

Low rates of helmet use among riders were noted in several studies, which may be linked to the high prevalence of head injuries following electric scooter-associated trauma. Moreover, one study noted a protective effect of helmets on craniofacial injuries suggesting many of these injuries may be preventable. ${ }^{13}$ This assertion is supported by a large body of work showing the effectiveness of helmets in preventing head injuries in cyclists. ${ }^{44}$ 
Table 5 Key findings of selected literature: injury circumstances

\begin{tabular}{|c|c|c|c|c|c|c|c|c|}
\hline \multirow[b]{2}{*}{ Study } & \multicolumn{2}{|c|}{ Mechanism (\%) } & \multirow[t]{2}{*}{ Location (\%) } & \multicolumn{3}{|c|}{ Helmet use (\%) } & \multicolumn{2}{|r|}{ Substance use (\%) } \\
\hline & Single user & Collision * & & Yes & No & Unknown & Alcohol & Other \\
\hline \multicolumn{9}{|c|}{ Peer-reviewed publications } \\
\hline Trivedi et al $2019^{16}$ & 88 & 9 & & 4 & 32 & 63 & 5 & \\
\hline Störmann et al $2020^{15}$ & 92 & 8 & & 1 & & & & \\
\hline Mitchell et al $2019^{13}$ & 69 & 31 & & 46 & 20 & 30 & & \\
\hline Liew et al $2020^{28}$ & & & & 6 & & & & \\
\hline Kobayashi et al $2019^{12}$ & & & & 7 & 88 & 5 & 48 & Positive toxicology screen: 30 \\
\hline Brownson et al $2019^{11}$ & 97 & 3 & & 2 & 19 & 79 & 13 & \\
\hline Blomberg et al $2019^{4}$ & 91 & 9 & & 4 & 55 & 41 & 37 & \\
\hline Beck et al 2019 & & & & 2 & 19 & 79 & 13 & \\
\hline Bauer et al $2020^{10}$ & 97 & 3 & & & & & 49 & \\
\hline Puzio et al $2020^{14}$ & & & & $0+$ & - & & 33 & \\
\hline Badeau et al $2019^{32}$ & & & Sidewalk: 44 & & & & & \\
\hline \multicolumn{9}{|l|}{ Namiri et al $2020^{3}$} \\
\hline \multicolumn{9}{|l|}{ Aizpuru et al $2019^{9}$} \\
\hline Dhillon et al $2020^{27}$ & 34 & 58 & & 18 & 71 & 11 & 24 & \\
\hline English et al $2020^{18}$ & 85 & 15 & $\begin{array}{l}\text { Street: } 71 \\
\text { Sidewalk: } 3 \\
\text { Unknown: } 26\end{array}$ & 1.6 & & & 11 & \\
\hline \multicolumn{9}{|l|}{ Farley et al $2020^{47}$} \\
\hline Bresler et al $2019^{23}$ & & & & 5 & 10 & 85 & & \\
\hline Yarmohammadi et al $2020^{20}$ & & & & $0 \dagger$ & & & 74 & \\
\hline Faraji et al $2020^{21}$ & & & & & & & 46 & Cannabis: 7 \\
\hline Trivedi et al $2019^{22}$ & & & & $0 \dagger$ & & & 18 & \\
\hline Hennocq $2020^{24}$ & & & Sidewalk: 31 & 12 & & & 49 & Other substance: 12 \\
\hline \multicolumn{9}{|l|}{ Kim et al $2020^{25}$} \\
\hline Oksanen et al $2020^{26}$ & & & & 17 & 0 & 83 & 91 & \\
\hline Shiffler et al $2020^{19}$ & 97 & 3 & & 12 & 1 & 87 & 12 & Other substance: 4 \\
\hline Siow et al $2020^{29}$ & & & & 3 & & & 27 & \\
\hline Ishmael et al $2020^{33}$ & 89 & 11 & & & & & & \\
\hline \multicolumn{9}{|l|}{ Nellamattathil and Amber $2020^{31}$} \\
\hline Mayhew and Bergin $2019^{30}$ & & & & & & 84 & & \\
\hline \multicolumn{9}{|c|}{ Grey literature (non-journal reports) } \\
\hline \multicolumn{9}{|l|}{ Pearson et al $2019^{35}$} \\
\hline \multicolumn{9}{|l|}{ Hojjat et al $2019^{36}$} \\
\hline \multicolumn{9}{|l|}{ Beck et al $2020^{37}$} \\
\hline \multicolumn{9}{|l|}{ Chang and Diamond $2019^{38}$} \\
\hline Allen et al $2019^{39}$ & 85 & 10 & & 2 & & & & \\
\hline Sedor and Caswell $2019^{40}$ & 94 & 6 & & 3 & & & 24 & \\
\hline Austin Public Health Unit $2019^{17}$ & & & $\begin{array}{l}\text { Street: } 55 \\
\text { Sidewalk: } 33\end{array}$ & 1 & & & 29 & \\
\hline \multicolumn{9}{|l|}{ City of Santa Monica 2019} \\
\hline Cicchino et al $2020^{34}$ & 69 & 28 & $\begin{array}{l}\text { Sidewalk: } 57 \\
\text { Road: } 24 \\
\text { Off-road: } 10 \\
\text { Bike lane: } 8 \\
\text { Other: } 2\end{array}$ & 2 & & & & \\
\hline
\end{tabular}

${ }^{*}$ Collision with another road user.

tDid not report unknown helmet status.

This evidence is relevant for many cities that are considering helmet laws for electric scooter users. ${ }^{6}$ A 2019 study in Brisbane, Australia found that previously low rates of helmet use increased to $64 \%$ among electric scooter riders following the introduction of a mandatory helmet law. ${ }^{45}$ More broadly, helmet use among electric scooter riders should be promoted in public health messaging as an effective means to reduce the high incidence of head injuries.

While the majority of electric scooter riders were injured in single road user events, a considerable portion were injured through collisions with other vehicles. Moreover, in some cases, cyclists and pedestrians were injured through collisions with electric scooter riders. These findings may in part be due to the scooters' high speeds and small size allowing them to be used on different types of road infrastructure. ${ }^{2}$ The five studies that reported location found that streets and sidewalks were common locations where these events occurred. ${ }^{1718243234}$ This suggests that policies restricting electric scooter use to specific road infrastructure such as bicycle lanes should be considered as both devices operate at similar speeds. ${ }^{6}$

Multiple findings highlight a large healthcare burden in cities where electric scooters are popular. For instance, although the 
majority of patients seen in the emergency department were discharged home, a considerable portion required admission to hospital. Moreover, over two-thirds of patients (68.9\%) required at least one procedure during their emergency department visit. These findings are supported by a New Zealand study which found that the introduction of electric scooters had a large impact on regional healthcare costs. ${ }^{46}$ This may be of particular interest to cities considering the adoption of shared electric scooter schemes, as the introduction of such services may increase the demand of already-stretched emergency services. ${ }^{47}$

\section{Limitations}

Our findings are affected by the limitations of the included literature. The majority of included studies were retrospective case series in design that only reported on clinically relevant variables present in medical charts or databases. Additionally, most studies only reported the clinical course of the patients' emergency department visits; information on long-term health outcomes is lacking. Metrics used to report important factors such as injury distribution and severity were heterogenous across studies, limiting the scope of comparisons. Factors relating to the circumstances of the injury such as the location of the event and substance use were inconsistently reported, while helmet use was difficult to ascertain due to high rates of unknown helmet status.

\section{Recommendations for future research}

The three studies with prospective observational designs benefitted from emergency department clinicians consistently documenting circumstances of the incidents as well as patients' clinical course and outcome. ${ }^{15}$ Future research on electric scooter trauma would benefit from a similar prospective observational design with an emphasis on using standard metrics such as ICD-10 body regions for reporting injury distribution, and ISS or Abbreviated Injury Scale for injury severity. Such research would provide better evidence on the injury patterns and severity of electric scooter-associated trauma. Details of the injury circumstance, such as time of day, road infrastructure (eg, sidewalk, roadway, bike path), involvement of other road users, and contributory factors should be systematically collected in order to identify modifiable risk factors for electric scooter injuries. Similarly, as electric scooter use increases, it is important for injury surveillance databases to capture electric scooter injuries, including injuries to other road users resulting from collisions with electric scooters. Moreover, as many urban areas lack legislation mandating the use of protective equipment by electric scooter riders, future evaluations of interventions for preventing electric scooter injuries, especially measures to increase helmet use, will help inform policy decisions.

\section{CONCLUSION}

While electric scooters are a convenient mode of inner-city transportation, they leave riders vulnerable to traumatic injuries. This review suggests that the head, upper extremities and lower extremities are particularly vulnerable in electric scooter trauma, while injuries to the chest and abdomen are less common. Notably, the low rates of helmet use reported among injured electric scooter users, and high rates of head injuries suggest the need for interventions to increase helmet use in this group of road users. Our findings also highlight the large burden placed on emergency departments by this popular mode of transportation. Most electric scooter studies to date have been retrospective case series. Future work should prospectively collect standardised data that include information on the context of the

\section{What is already known on this subject}

- The rise in electric scooter use has led to an increase in the incidence of electric scooter-associated injuries, which have involved both riders and other road users.

- Studies of electric scooter injuries have been conducted in different settings using a range of methodologies.

\section{What does this study add}

- Review findings suggest that the head, the upper extremities and the lower extremities are particularly vulnerable in electric scooter injuries.

- Most electric scooter injuries involve a single user, with falls being the most common mechanism of injury.

- Low rates of helmet use among electric scooter riders were noted in multiple studies, potentially leaving riders more vulnerable to head injuries.

injury events and electric scooter usage patterns, as well as key clinical variables. Finally, research on interventions to prevent electric scooter injuries will be important to address this growing concern and advance public health.

Contributors The study was conceived of by MT. JRB and HC provided methodological guidance. MT and SM conducted the systematic review with guidance from JRB, HC and LKS. All authors contributed to the writing of the manuscript and reviewed and approved the final version of the manuscript.

Funding The authors have not declared a specific grant for this research from any funding agency in the public, commercial or not-for-profit sectors.

Competing interests None declared.

Patient consent for publication Not required.

Ethics approval This study consisted solely of a literature review and requirement for ethics review was waived by the University of British Columbia Research Ethics Board.

Provenance and peer review Not commissioned; externally peer reviewed. Data availability statement Data are available in public, open access repositories.

Supplemental material This content has been supplied by the author(s). It has not been vetted by BMJ Publishing Group Limited (BMJ) and may not have been peer-reviewed. Any opinions or recommendations discussed are solely those of the author(s) and are not endorsed by BMJ. BMJ disclaims all liability and responsibility arising from any reliance placed on the content. Where the content includes any translated material, BMJ does not warrant the accuracy and reliability of the translations (including but not limited to local regulations, clinical guidelines, terminology, drug names and drug dosages), and is not responsible for any error and/or omissions arising from translation and adaptation or otherwise.

Open access This is an open access article distributed in accordance with the Creative Commons Attribution Non Commercial (CC BY-NC 4.0) license, which permits others to distribute, remix, adapt, build upon this work non-commercially, and license their derivative works on different terms, provided the original work is properly cited, appropriate credit is given, any changes made indicated, and the use is non-commercial. See: http://creativecommons.org/licenses/by-nc/4.0/.

\section{ORCID iD}

Jeffrey R Brubacher http://orcid.org/0000-0002-4866-4231

\section{REFERENCES}

1 Choron RL, Sakran JV. The integration of electric Scooters: useful technology or public health problem? Am J Public Health 2019;109:555-6.

2 Todd J, Krauss D, Zimmerman J. Behavior of electric scooter operators in naturalistic environments: SAE technical paper series. , 2019: 01, 10007.

3 Namiri NK, Lui H, Tangney T, et al. Electric scooter injuries and hospital admissions in the United States, 2014-2018. JAMA Surg 2020;155:357-9. 
4 Blomberg SNF, Rosenkrantz OCM, Lippert F, et al. Injury from electric scooters in Copenhagen: a retrospective cohort study. BMJ Open 2019;9:e033988.

5 Beck S, Barker L, Chan A, et al. Emergency department impact following the introduction of an electric scooter sharing service. Emerg Med Australas 2020;32:409-15.

6 Gössling S. Integrating e-scooters in urban transportation: problems, policies, and the prospect of system change. Transp Res D Transp Environ 2020;79:102230.

7 Tricco AC, Lillie E, Zarin W, et al. PRISMA extension for scoping reviews (PRISMA-SCR): checklist and explanation. Ann Intern Med 2018;169:467-73.

8 National Heart, Lung, and Blood Institute. Study quality assessment tools, 2019. Available: https://www.nhlbi.nih.gov/health-topics/study-quality-assessment-tools

9 Aizpuru M, Farley KX, Rojas JC, et al. Motorized scooter injuries in the era of scootershares: a review of the National electronic surveillance system. Am J Emerg Med 2019:37:1133-8.

10 Bauer F, Riley JD, Lewandowski K, et al. Traumatic injuries associated with standing motorized Scooters. JAMA Netw Open 2020;3:e201925.

11 Brownson AB, Fagan PV, Dickson S, et al. Electric scooter injuries at Auckland City Hospital. N Z Med J 2019;132:62-72.

12 Kobayashi LM, Williams E, Brown CV, et al. The e-merging e-pidemic of e-scooters. Trauma Surg Acute Care Open 2019;4:e000337.

13 Mitchell G, Tsao H, Randell T, et al. Impact of electric scooters to a tertiary emergency department: 8-week review after implementation of a scooter share scheme. Emerg Med Australas 2019;31:930-4.

14 Puzio TJ, Murphy PB, Gazzetta J, et al. The electric scooter: a surging new mode of transportation that comes with risk to riders. Traffic Inj Prev 2020;21:175-8.

15 Störmann P, Klug A, Nau C, et al. Characteristics and injury patterns in electricscooter related accidents-a prospective two-center report from Germany. J Clin Med 2020;9:1569-e69.

16 Trivedi TK, Liu C, Antonio ALM, et al. Injuries associated with standing electric scooter use. JAMA Netw Open 2019;2:e187381.

17 Austin Public Health Unit. Dockless electric Scooter-Related injuries study. Austin Texas, 2019.

18 English KC, Allen JR, Rix K, et al. The characteristics of dockless electric rental scooterrelated injuries in a large U.S. City. Traffic Inj Prev 2020;21:476-81.

19 Shiffler K, Mancini K, Wilson M, et al. Intoxication is a significant risk factor for severe Craniomaxillofacial injuries in standing electric scooter accidents. J Oral Maxillofac Surg 2020;S0278-2391(20:31194-0.

20 Yarmohammadi A, Baxter SL, Ediriwickrema LS, et al. Characterization of facial trauma associated with standing electric scooter injuries. Ophthalmology 2020;127:988-90

21 Faraji F, Lee JH, Faraji FLee JH, MacDonald B, et al. Electric scooter craniofacial trauma. Laryngoscope Investig Otolaryngol 2020;5:390-5.

22 Trivedi B, Kesterke MJ, Bhattachariee R, et al. Craniofacial injuries seen with the introduction of Bicycle-Share electric Scooters in an urban setting. J Oral Maxillofac Surg 2019;77:2292-7.

23 Bresler AY, Hanba C, Svider P, et al. Craniofacial injuries related to motorized scooter use: a rising epidemic. Am J Otolaryngol 2019;40:662-6.

24 Hennocq Q, Schouman T, Khonsari RH, et al. Evaluation of electric scooter head and neck injuries in Paris, 2017-2019. JAMA Netw Open 2020;3:e2026698.

25 Kim M, Lee S, Ko DR, et al. Craniofacial and dental injuries associated with stand-up electric scooters. Dent Traumatol 2020;00:1-5
26 Oksanen E, Turunen A, Thorén H. Assessment of Craniomaxillofacial injuries after electric scooter accidents in Turku, Finland, in 2019. J Oral Maxillofac Surg 2020;78:2273-8.

27 Dhillon NK, Juillard C, Barmparas G, et al. Electric scooter injury in southern California trauma centers. J Am Coll Surg 2020;231:133-8.

28 Liew YK, Wee CPJ, Pek JH. New peril on our roads: a retrospective study of electric scooter-related injuries. Singapore Med J 2020;61:92-5.

29 Siow MY, Lavoie-Gagne 0, Politzer CS, et al. Electric scooter orthopaedic injury demographics at an urban level I trauma center. J Orthop Trauma 2020;34:e424-9.

30 Mayhew LJ, Bergin C. Impact of e-scooter injuries on emergency department imaging. J Med Imaging Radiat Oncol 2019;63:461-6.

31 Nellamattathil $M$, Amber I. An evaluation of scooter injury and injury patterns following widespread adoption of E-scooters in a major metropolitan area. Clin Imaging 2020;60:200-3.

32 Badeau A, Carman C, Newman M, et al. Emergency department visits for electric scooter-related injuries after introduction of an urban rental program. Am J Emerg Med 2019;37:1531-3.

33 Ishmael CR, Hsiue PP, Zoller SD, et al. An early look at operative orthopaedic injuries associated with electric scooter accidents: bringing high-energy trauma to a wider Audience. J Bone Joint Surg Am 2020;102:e18

34 Cicchino JB, Kulie PE, McCarthy ML. Injuries related to electric scooter and bicycle use in a Washington, DC emergency department, 2020. Available: https://www.iihs.org/ api/datastoredocument/bibliography/2215

35 Pearson T, Mitchell G, Hacking C. Injury patterns in e-scooter trauma. J Med Imaging Radiat Oncol 2019;63:69.

36 Hojjat H, Cowan B, Lucas J. Gliding into the emergency department: scooter injuries to the head and neck. Otolaryngol Head Neck Surg 2019;161.

37 Beck S, Barker L, Chan A. Emergency department impact of injuries associated with standing electric scooters - A retrospective case series. Emerg Med Australas 2020;32:66-7.

38 Chang A, Diamond P. Traumatic brain injury related to Stand-up motorized scooter usage. Arch Phys Med Rehabil 2019;100:e207.

39 Allen JR, English KC, Rix K, et al. 262 epidemiology of Dockless electric Rental scooter injuries. Ann Emerg Med 2019;74:S103.

40 Sedor A, Caswell N. Shared e-Bike and e-Scooter Mid-Pilot report (city of Calgary) 2019. Available: https://www.calgary.ca/content/dam/www/transportation/tp/ documents/cycling/cycling_strategy/shared-e-scooter-and-e-bike-mid-pilot-councilreport.pdf

41 City of Santa M. Shared mobility pilot program summary report, 2019: 71p.

42 Nathanson BH, Ribeiro K, Henneman PL. An analysis of US emergency department visits from falls from skiing, Snowboarding, Skateboarding, Roller-Skating, and using Nonmotorized Scooters. Clin Pediatr 2016;55:738-44.

43 Chiu J, Robinovitch SN. Prediction of upper extremity impact forces during falls on the outstretched hand. J Biomech 1998;31:1169-76.

44 Olivier J, Creighton P. Bicycle injuries and helmet use: a systematic review and metaanalysis. Int J Epidemiol 2017:46:1:278-92.

45 Haworth NL, Schramm A. Illegal and risky riding of electric scooters in Brisbane. Med Aust 2019:211:412-3.

46 Bekhit MNZ, Le Fevre J, Bergin CJ. Regional healthcare costs and burden of injury associated with electric scooters. Injury 2020;51:271-7.

47 Farley KX, Aizpuru M, Wilson JM, et al. Estimated incidence of electric scooter injuries in the US from 2014 to 2019. JAMA Netw Open 2020;3:e2014500. 